The Japanese Journal of Animal Psychology, 50, 2, 213-220 (2000)

Original Article

\title{
The effects of fixed sequence of US intensities and CS-US correlation on licking suppression in rats
}

\author{
NOBUTAKA DOE ${ }^{1}$ \\ Kwansei Gakuin University
}

\begin{abstract}
Two experiments investigated the effects of shock (US) sequence and correlation between signals (CSs) and shocks (USs) on contextual fear with conditioned licking suppression preparation. In both experiments, three shocks of different intensities were presented in fixed or random order. These shocks were correlated or uncorrelated with three different tones in Experiment 1, and with light, tone, and white noise in Experiment 2. In Experiment 1, the basal licking behavior was more suppressed in the groups with random shock sequence than in the groups with fixed shock sequence, regardless of combination of signals with shocks. Whereas in Experiment 2, the basal licking suppression was greater when signals and shocks were uncorrelated than when they were correlated, regardless of shock sequence. These results suggest that the factor to attenuate contextual fear assessed by licking suppression varies depending on the degree of distinctiveness among signals.
\end{abstract}

Key words : information hypothesis, US sequence, CS-US correlation, licking suppression, rats.

Given a situation in which inescapable and unavoidable shocks are presented, rats typically prefer signaled shock condition to unsignaled shock condition (e. g., Arabian \& Desiderato, 1975; Badia \& Culbertson, 1972; Fanselow, 1980; Frankel \& Vom Saal, 1976; Furedy \& Biederman, 1976; Lockard, 1963). This phenomenon is known as the preference for signaled shock (PSS). Moreover,chronicfearelicited in thesignaled shock condition is less than that in the unsignaled shock condition. This chronic fear has been defined as contextual fear or a basal emotional level (BEL) (Imada \& Soga, 1971), and examined in many studies using various procedures and indexes: suppression of basal rate of bar-pressing (Seligman, 1968), suppression of basal rate of licking behavior (Imada \& Soga, 1971; Nageishi \& Imada, 1974), the amount of time spent for freezing (Bolles \& Collier, 1976), and the amount of stomach lesions produced (Seligman, 1968; Seligman \& Meyer, 1970; Weiss, 1970).

1 Present address: e-mail : doe@kwansei.ac.jp Dept. of Psychology, Kwansei Gakuin University, Nishinomiya $602-8501$
To account for the PSS and the attenuation of the chronic fear in the signaled shock condition, some mechanisms have been proposed: the preparatory-response hypothesis, the safetysignal hypothesis, and the information hypothesis. According to the preparatory-response hypothesis, the signal permits animals to make some type of preparatory response, either peripheral or central, which serves to reduce the experienced aversiveness of theshock (e.g.,Perkins, 1968). However, what the preparatory response is, as of yet, is unknown. The safety-signal hypothesis emphasizes non-signal periods as the crucial factor. Animals prefer the signaled shock condition because shock never occurs during non-signal periods and, consequentially, non-signal periods generate a much lower level of fear than is elicited by comparable non-shock periods in the unsignaled shock condition. Signal offset initiates a discriminable shock-free period (e. g., Badia \& Culbertson, 1972; Seligman,1968). Theinformation hypothesis interprets the PSS as indicating that animals placed in an aversive situation will make every effort to get and utilizeinformation to reduce their chronic fear. The information provided by 
the signal may function as a reinforcer and thus strengthen the response of animals to contact with the signaled shock condition (e. g., Berlyne, 1960). In this research, we pay close attention to the information hypothesis. However, these three processes are not necessarily observed exclusively. In a way, they may be present concurrently.

Some studies have shown evidence that support the information hypothesis or the preparatory-response hypothesis (e. g., Collier, 1977; D'Amato \& Safarjan, 1979; Marlin, Sullivan, Berk, \& Miller, 1979). For example, D'Amato and Safarjan (1979) found that, in a change-over bar-pressing situation, rats preferred the condition in which two different signals were correlated with two different shock durations as opposed to the condition in which signals and shocks were uncorrelated. Marlin et al. (1979) also reported that, given a two-compartment choice situation, rats showed preference for the compartment in which two different signals were correlated with two different shock intensities to the compartment in which these were uncorrelated. These two studies demonstrated that rats placed in an inescapable-shock situation prefer the condition in which signals provide information of shock properties. From this viewpoint, the PSS can be regarded not only as "preference for signaled occurrence of shock" but also as "preference for signaled properties of shock".

Fujii, Uchida, and Imada (1994) investigated the effects of precise information about shock intensities on contextual fear assessed by licking suppression. Shocks of two different intensities were presented with signals of two different modalities. The signal modalities and the shock intensities were correlated for Group C (Correlated), but they were not for Group UC (Uncorrelated). The basal rate of licks was suppressed more in Group UC than in Group C, suggesting that fear of the situation was reduced by having precise information about shocks.

However, the signals of shocks are not the only information sources for occurrence and properties of the shocks. Presenting different shocks in fixed order may provide information about shocks (e. g., Imada \& Okamura, 1975; Imada, Sugioka, Ohki, Ninohira, \& Yamazaki, 1978). For example, Imada and Okamura (1975) presented six shocks with a FT 45-s schedule in every session. The strong and weak shocks occurred alternately. A clearpatterningin lickingsuppression wasshown. This finding suggests that rats can utilize not only signals preceding shocks but also shock sequence as a cue to predict occurrence and properties of shocks.

If the factor of shock sequence is added to the correlation-uncorrelation paradigm used by D'Amato and Safarjan (1979), Marlin et al. (1979), and Fujii et al. (1994), it is possible to operate the amount of information hierarchically and verify the validity of the information hypothesis.

In this research, the effects of fixed shock sequenceand correlation between signals and shock$\mathrm{s}$ on contextual fear were investigated.

\section{Experiment 1}

In Experiment 1, under the situation in which three electric shocks of different intensities were presented with three different signals, the effects of shock sequence and the correlation between signals and shockson contextualfear assessed bylicking suppression was explored. It is thought that contextual fear is attenuated in cases where the sequence of shocks is fixed even though signals are not correlated with shocks.

\section{Method}

Subjects Thesubjects were 48 experimentally naive male Wistar rats. They were approximately 60 days old and weighed $274.0 \mathrm{~g}$ on average (range: $215-298 \mathrm{~g}$ ), at the beginning of the experiment. They were kept in stainless-steel cages individually in a room maintained on a 12:12 light-dark cycle.Food was freely available throughout the experiment. Apparatus The apparatus consisted of six identical drinking boxes (seeKawai, Nishida, \& Imada, 1998). Each drinking box had a grid floor, a transparent ceiling, and white walls $(20 \mathrm{~cm}$ long $\times 10$ $\mathrm{cm}$ wide $\times 15 \mathrm{~cm}$ high, inner dimensions). On the lower part of the wall, at one end of the box, was a hole (12 $\mathrm{mm}$ in diameter) through which a rat could lick a drinking tube. Contact with the drinking tube was detected by using a low-current circuit between the tube and the grid floor. The boxes were placed on a round table, in a way that each 
box was separated from the adjacent two by an equal distance. A fan was attached directly below the table to provide a masking noise of approximately $65 \mathrm{~dB}(\mathrm{C})$. The apparatus was illuminated by indirect lighting, which shed light of approximately $10 \mathrm{~lx}$ into each box. For the unconditioned stimuli (USs), three kinds of electric shocks that differed in intensity $(75 \mathrm{~V}, 100 \mathrm{~V}$, and $125 \mathrm{~V})$ were delivered to the subject from an alternating current shock source to the grid floor through a $250-\mathrm{k}$ $\Omega$ current-limiting resistor put in series with the rat. The duration of these USs was $0.7 \mathrm{~s}$. For the conditioned stimuli (CSs), threekinds of tones that differed in frequency $(1000 \mathrm{~Hz}, 1500 \mathrm{~Hz}$, and 2000 $\mathrm{Hz}$ ) were used. The CSs were delivered from a speaker attached $95 \mathrm{~cm}$ directly above the center of the table, approximately $85 \mathrm{~dB}(\mathrm{C})$. The duration of these CSs was 10s.

Procedure Prior to the start of the experiment, all subjects were tamed by handling for five minutes in each of three consecutive days. From the third day of handling, all subjects were placed in a 15-minute-per-day watering schedule, which was maintained throughout the experiment. Experiment 1 consisted of the next two phases.

Phase 1 (10 days): Preliminary training of drinking

The purpose of this phase was to build steady licking behavior. All the subjects were trained in the drinking boxes for five minutes daily to drink water by licking at the tip of the drinking tube. Aftereach session, thesubjects were returned to their cages, and 10 minutes later, they were allowed to drink water for 10 minutes. On the last day, the subjects were divided into four groups of 12 rats each matched by the mean number of licks persession and the mean body weight for the last three days of this phase. These groups were labeled as Group C-F (Correlated-Fixed), Group C-R (Correlated-Random), Group UC-F (Uncorrelated-Fixed), and Group UC-R (Uncorrelated-Random).

Phase2(12days):Conditioning Threetypes offear conditioning trials were given in a daily session of five minutes, superimposed upon licking baseline behavior. In each trial, a US immediately followed a CS. The inter-trial interval (US-offset-CS-onset interval) was changed randomly, and was $66.7 \mathrm{~s}$ on average (range: $40-90 \mathrm{~s}$ ).

This experiment was based on a $2 \times 2$ facto- rial design. The first factor was the combination of CSs and USs (correlated vs. uncorrelated). For thetwogroups of correlated condition (Groups C-F and $\mathrm{C}-\mathrm{R}$ ), frequencies of tones used as CSs and intensities of USs werecorrelated. The CS1,CS2, and CS3 were always followed by weak-, middle-, and strong-US, respectively.In each group, theCS1 was a $1000 \mathrm{~Hz}$-tone and the CS3 was a $2000 \mathrm{~Hz}$-tone for half of the subjects, and vice versa for the other half. The CS2 was a $1500 \mathrm{~Hz}$-tone for all the subjects. On the other hand, for the two groups of uncorrelated condition (Groups UC-F and UC-R), the combination of CSs and USs were changed every session. Three different USs at an equal frequency followed each CS over the conditioning phase. The second factor was the US sequence (fixed vs. random). For the two groups of fixed condition (Groups C-F and UC-F), three conditioning trials were presented in fixed order (weak-middle-strong) in each session. For the two groups of random condition (Groups C-R and UC-R), the US sequence was changed every session. The US sequences used for the initial six days of this phase were SMW, WSM, MWS, MSW, SWM, and WMS (W, M, and Srepresenting weak-, middle-, and strong-US). This series of sequences was repeated twice.

Group C-F was allowed to utilize not only the CSs but also the US sequence as a cue to predict US intensity. Groups C-R and UC-F were given either of them. Therefore, information about US intensity was given for these three groups. In contrast, only information about US occurrence was provided for Group UC-R.

\section{Results and Discussion}

\section{Baseline Licking Suppression (Contextual Fear)}

The baseline number of licks for a rat in each session (per $270 \mathrm{~s}$ ) was calculated by subtracting thenumberoflicksduring thepresentation of three CSs from the total number of licks in that session. Figure 1 shows the mean baseline number of licks during the conditioning phase in blocks of three days. The Pre indicates 90 percent of the total number of licks in a session (per $270 \mathrm{~s}$ ) for the last three days of the preliminary training phase.

As can be seen in Figure 1, throughout the conditioning phase, there were more licks in Groups 


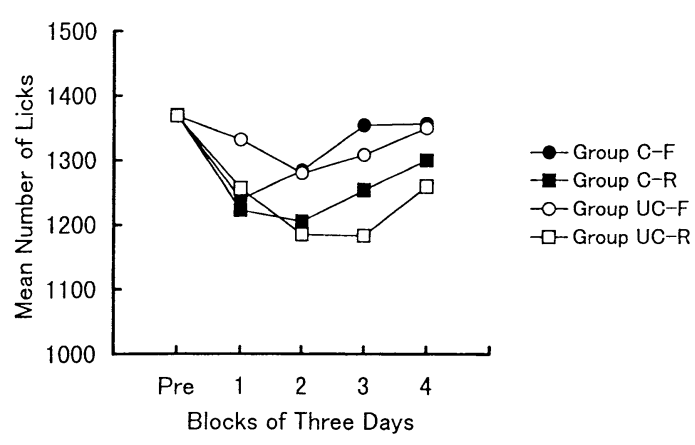

Figure 1. Mean baseline number of licks (per 270 s) of four groups in Experiment 1.

C-F and UC-F, in which thethreedifferentUSs were presented in fixed order, than Groups C-R and UC-R, in which the USs were presented randomly. The number of licks in Groups C-F and UC-F recovered to the Pre level gradually after an initial decrease, and a marked difference between these two groups was not observed. The number of licks in Groups C-R and UC-R changed concurrently, although a slight difference between the groups was seen in blocks 3 and 4 .

A 2 (CS-US combination: correlated vs. uncorrelated) $\times 2$ (US sequence: fixed vs. random $) \times 4$ (block) ANOVA revealed significant main effects of US sequence $(F(1,44)=5.06, p<.05)$ and block $(F(3,132)=2.73, p<.05)$. The main effect of CS-US combination $(F<1)$ and all the interactions were not significant. The results of this statistical analysis confirm what has been described above. Namely, it was suggested that the presentation of USs in fixed order attenuated the suppression of basal rate of licks. The effect of CS-US correlation observed in Fujii et al. (1994) was not reconfirmed in Experiment 1.

\section{CS Suppression (US intensity anticipation)}

As an index of conditioned suppression during presentation of each CS, a conventional suppression ratio (SR, Annau \& Kamin, 1961) was employed. The SR was calculated by B / $(A+B)$, where $B$ represents the number of licks during the $\mathrm{CS}$, and $\mathrm{A}$ the number of licks in the $10 \mathrm{~s}$ immediately preceding the CS. The SR of 0.5 indicates nosuppression, and that ofzeroindicatescomplete suppression.

Figure 2 shows the mean SRs for each of four groups on weak-, middle-, and strong-US trials in
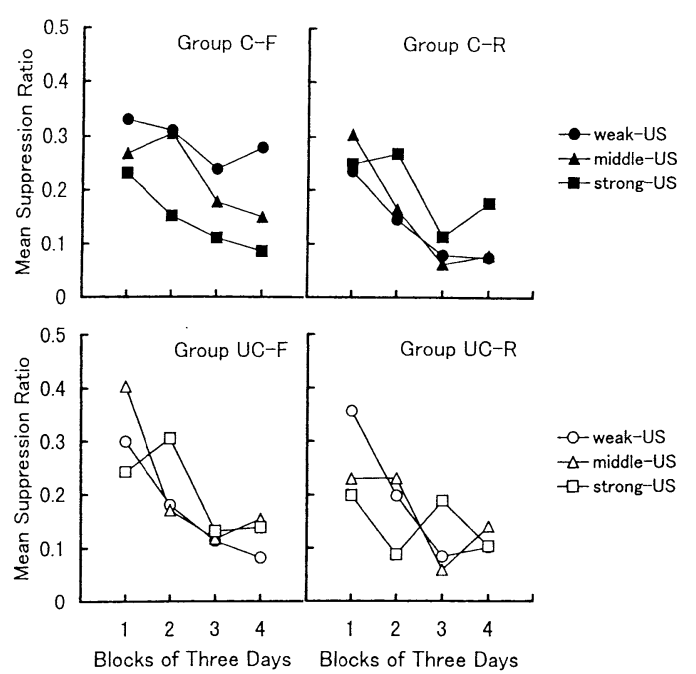

Figure 2. Mean suppression ratio for fourgroups in Experiment 1.

blocks of three days. Although suppression to CSs was evident for all groups, a distinct correlation between suppression level and different US intensities was observed only in Group C-F. Group C-F showed the greatest suppression to the CS3 followed by strong-US and the weakest suppression to the CS1 with weak-US. In Group UC-R, there was no reason to expect suppression to differ among the three types of trials because the USs were not correlated with the CSs, and the US sequence was changed across sessions. The experimental data for Group UC-R confirmed this prediction. However, unexpected results were obtained in Groups C-R and UC-F. Although these two groups were permitted to utilize either US sequence or CS-US correlation as a cue to predict USintensity, they did notshow the differential suppression similar to that observed in Group C-F.

A 2 (CS-US combination: correlated vs. uncorrelated) $\times 2$ (US sequence: fixed vs. random $) \times 3$ (US intensity) $\times 4$ (block) ANOVA conducted using the data summarized on Figure 2 yielded significant main effects of US sequence $(F(1,42)=$ $5.97, p<.05)$ and block $(F(3,126)=55.48, p<.01)$. The interactions of US sequence $\times$ US intensity $(F(2,84)=6.03, p<.01)$, CS-UScombination $\times$ US sequence $\times \operatorname{USintensity}(F(2,84)=22.22, p<.01)$, and CS-US combination $\times$ US sequence $\times$ US intensity $\times$ block $(F(6,252)=8.31, p<.01)$ were all significant. Subsequent Fisher's Protected LSD 
revealed the significant differences in the $\mathrm{SR}$ as follows: weak- $>$ middle- $>$ strong-US trial in Group C-F; strong-US trial $>$ the other two US trials in Group C-R. The differential suppression in Group C-F suggests that theinformation about US intensity was well assimilated by the subjects of this group. Additionally, because Group C-R showed the reliably weaker suppression on the strong-US trial than on the other trials, although it is not definitive, it may be concluded, that the subjects distinguished three kinds of tones used as CSs. As for Groups UC-F and UC-R, any difference that could be explained logically did not prove to be significant in each block.

Findings in Experiment 1 may be summarized as follows: (a) It is not necessarily true that the CS-US correlation regularly brings about a decrease of contextual fear and differential suppression todifferenttrials.(b)Thefixed shocksequence has been found to have an effect on a decrease of contextual fear.

\section{Experiment 2}

Experiment 1 clearly demonstrated that the fixed US sequence attenuated the baseline licking suppression. Theeffect of correlation between CSs and USs was not revealed. This result was inconsistent with Fujii et al. (1994). What factors are responsible for the discrepancy between these two findings? TheCSmaterials used in Fujiiet al.(1994) were different from those used in Experiment 1.

Experiment 2 was carried out with the identical design as Experiment 1, except that the light and tone used in Fujii et al. (1994) were employed as CSs. The purpose of Experiment 2 is to examine the effect of CS-US correlation under the situation in which three kinds of USs were presented.

\section{Method}

Subjects Thesubjectswere 48 experimentally naive male Wistar rats, approximately 60 days old, and their mean body weight was $267.1 \mathrm{~g}$ (range: $245-288 \mathrm{~g}$ ) at the beginning of the experiment. They were kept under the same conditions as Experiment 1.

Apparatus The apparatus was identical to that used in Experiment 1. As CSs, the light (126 lx),
$1500 \mathrm{~Hz}$-tone (approximately $85 \mathrm{~dB}(\mathrm{C})$ ), and white noise (approximately $85 \mathrm{~dB}(\mathrm{C})$ ) were used. Illumination was provided by using a $100 \mathrm{~W}$ electric bulb fixed $85 \mathrm{~cm}$ directly above the drinking boxes.

Procedure Instead of the $1000 \mathrm{~Hz}$ - and 2000 $\mathrm{Hz}$-tones used in Experiment 1, the light and white noisewereused.Pre-experimental treatments, watering schedule, and the other procedural details were also same as in Experiment 1.

\section{Results and Discussion}

\section{Baseline Licking Suppression (Contextual Fear)}

Figure 3 shows the mean baseline number of licks for four groups. As in Experiment 1, the baseline suppression of Group UC-R was the greatest of all, and that of Group C-F was extremely weak. However, Group UC-F showed greater suppression than Group C-R.

A 2 (CS-US combination: correlated vs. uncorrelated) $\times 2$ (US sequence: fixed vs. random $) \times 4$ (block) ANOVA was conducted for the data summarized on Figure 3. Themain effect of CS-UScombination was significant $(F(1,44)=4.38, p<.05)$, butthemain effects of USsequence $(F<1)$ and block $(F(3,132)=1.59)$ were not significant. All the interactions did not prove to be significant. The results of this statistical analysis indicate that the correlation ofCSswithUSsattenuated thebaseline licking suppression. This finding is in agreement with the account by Fujii et al. (1994).

\section{CS Suppression (US intensity anticipation)}

Figure 4 shows the mean SRs of weak-, middle-, and strong-US trials in each group in blocks of three days. Clear differential suppression was

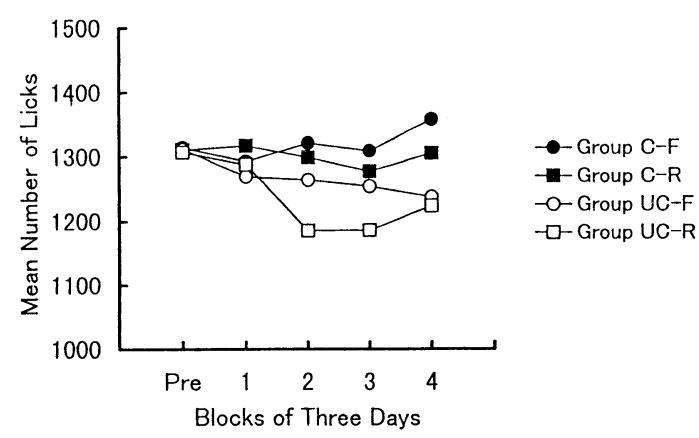

Figure 3. Mean baseline number of licks (per 270 s) of four groups in Experiment 2. 

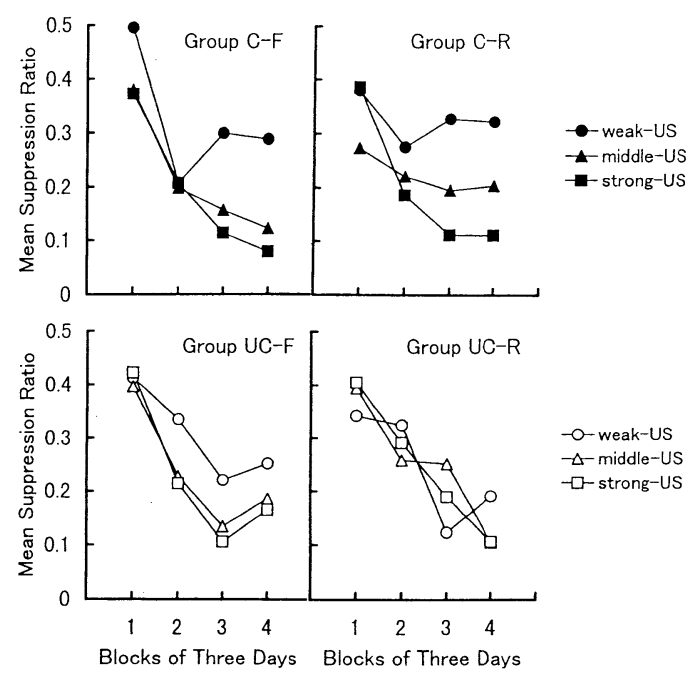

Figure 4. Mean suppression ratio for fourgroups in Experiment 2.

obtained in Groups C-F and C-R for which USs were correlated with CSs. In these groups, the greatest suppression was observed with the CS3 paired with strong-US, and the weakest suppression was observed with the CS1 paired with weak-US. Group UC-F also showed such a differentiation, but there was only a slight difference between strong- and middle-US trial.In Group UC-R, with neither CS-US correlation nor fixed US sequence, there was no reason to expect differential suppression. The experimental data for Group UC-R confirmed this prediction.

A 2 (CS-US combination: correlated vs. uncorrelated) $\times 2$ (US sequence: fixed vs. random $) \times 3$ (US intensity) $\times 4$ (block) ANOVA on the data shown in Figure 4 yielded significant main effects of US intensity $(F(2,88)=26.91, p<.01)$ and block $(F(3,132)=91.20, p<.01)$, and the interactions of CS-UScombination $\times \operatorname{USintensity}(F(2,88)=8.02$, $p<.01)$, CS-US combination $\times$ US sequence $\times$ block $(F(3,132)=2.92, p<.05)$, CS-US combination $\times$ US intensity $\times$ block $(F(6,264)=3.89$, $p<.01)$, and CS-UScombination $\times$ USsequence $\times$ USintensity $\times \operatorname{block}(F(6,264)=2.22, p<.05)$ were significant. Subsequent Fisher's Protected LSD revealed the significant differences in the $\mathrm{SR}$ as follows: weak- $>$ middle- $>$ strong-US trial in Group C-F and in blocks 2, 3, and 4 of Group C-R; weak-US trial $>$ the other two US trials in blocks 2 and 3 of Group UC-F. The differential suppres- sion in Groups C-F and C-R indicate that the information derived from the CS-US correlation was well assimilated by the subjects of these groups. On the other hand, it is supposed that Group UC-F that showed the correct anticipation only to weak-US trial did not utilize the information enough, which could be derived from the US sequence. For Group UC-R, any differentiation that could be explained logically did not prove to be significant in each block.

Based on the above conclusions, findings in Experiment 2 may be summarized as follows: (a) It is not necessarily true that the fixed US sequence regularly have an effect on a decrease of contextual fear. (b) The factor to facilitate the differential suppression by different trials was the CS-US correlation.

\section{General Discussion}

Experiments 1 and 2 had the following points in common: (a) One of the groups that showed the weakest baseline suppression was Group C-F, which was allowed to utilize both CS-US correlation and US sequence to predict US intensity. (b) Oneof thegroups that showed thegreatest baseline suppression was Group UC-R, which was not given any cue. These results suggest that giving precise information about shock moderates contextual fear, and are roughly consistent with the prediction deduced from the information hypothesis. Moreover, this research found that not only the CS-US correlation but also the sequence of USs could be utilized as an information source (Experiment 1 ). In this respect, this research succeeded in further developing the information hypothesis, which, until now, had been mainly examined with the correlation-uncorrelation paradigm.

Also, the differences between the two experiments lead to some useful findings. In Experiment 1 , as had already been mentioned above, regardless of the correlation of CSs with USs, the basal rate of licks was suppressed more in the groups with random US sequence than in the groups with fixed US sequence. This result suggests that the subjects would search and utilize the sequence of USs as a dominant cue to predict US intensity resulting in reducing their chronic fear. On the other hand, in Experiment 2, regardless of the US ar- 
rangement, the greater suppression of licks was shown in the groups in which three different USs were not correlated with three different CSs, as compared with the groups in which they were correlated. This result suggests that the subjects would search and utilize the CS-US correlation as a dominant cue. In Group C-R of Experiment 1, the utilization of the CS-US correlation was not confirmed although it was the only available information source. Similarly, in Group UC-F of Experiment 2 , the only available information source (US sequence) seems not to have been utilized. Therefore, the sequence of USs and the CS-US correlation were not always effective as a cue. It would be thought that these two information sources would have counteracted or excluded each other, without cooperating. When the subjects were motivated to seek and utilize one of the two information sources, it is thought that the search for the other information source is obstructed.

Theonly proceduraldifferencebetweenExperiments 1 and 2 wasthetypeofCSs. Theirregularities in cue utilization can be explained by examining the difference between them. The most reasonable interpretation of the difference of CSs might be the difference of distinctiveness (discriminability) among CSs. In Experiment 1, Group C-F showed a clear differentiation, but Group C-R did not show this. Because identical CS-US trials were given for these two groups, the same result would likely have been obtained in both groups. Group C-R of Experiment 2 showed a clear correlative differentiation as in Group C-F. It may be inferred that the reason for the failure to find such a differentiation in Group C-R of Experiment 1 might bethelow distinctiveness (discriminability) among the three kinds of tones used as CSs.

From what has been described above, it seems appropriate to conclude the following: (a) If it is difficult to distinguish CSs, rats utilize the US sequence as a main cue to predict US intensity. In this case, CSs might be merely signals for US occurrence. (b) If it is easy to distinguish CSs, rats utilize the properties of CSs as a main cue to predict occurrence and intensity of US. Therefore, the distinctiveness (discriminability) of CSs determined whether they would be utilized as a dominant cue.

The findings of this research cannot be ex- plained solely by the safety-signal hypothesis because all the subjects of Experiments 1 and 2 were observed under the same conditions, such as length of the discriminable shock-free periods. If only the length and discriminability of safety periods were the primary factors in determining the level of contextual fear, then under the situation of these experiments, differences in the level of contextual fear would not be observed. Once more, according to the preparatory-response hypothesis, although theresults of theseexperiments would not be inconceivable, they would beconsidered highly unlikely. Our findings suggest that accurate anticipation of US intensities has a close relationship to the actual effective lowering of contextual fear. In order to interpret the results of this study, according to the preparatory-response hypothesis, it is necessary to suppose that rats can exhibit each different preparatory-response according to at least threedifferent shock intensities.

In closing, in terms of interpreting that there is a definitive relationship between information about USs and a decrease in contextual fear, it is best to base these conclusions on the information hypothesis. However, as we gather more information, it is necessary to consider the factors that determine the dominant cue that is to be utilized.

Finally, I want to point out the relationship between the differentiation of SRs and the baseline suppression. As described in Fujii, et al. (1994), it is thought that the differentiation is the evidence of accurate anticipation of US intensities on different trials. To put it more plainly, the differentiation is the evidence of cue utilization. Actually, the baseline suppression was attenuated in Groups C-F and C-R of Experiment 2, which showed a clear differentiation. For these two groups, the utilization of information was confirmed by two indexes: the attenuation of baseline suppression and the differentiation. However, in Experiment 1, Group UC-F did not show a clear differentiation but showed the very weak baseline suppression. This result may be explained by assuming that the change of baseline licking behavior is a more sensitive index for detecting cue utilization than the differentiation of SRs. Although it is necessary to further research the validity of this assumption, the methods using contextual fear as an index of cue utilization may be effec- 
tive in further examining and developing the information hypothesis.

\section{References}

Annau, Z., \& Kamin, L. J. 1961 The conditioned emotional response as a function of the intensity of the US. Journalof Comparative andPhysiological Psychology, 54, $428-432$.

Arabian, J. M., \& Desiderato, O. 1975 Preference for signaled shock: A test of two hypotheses. Animal Learning \& Behavior, 3, $191-195$.

Badia, P., \& Culbertson, S. 1972 The relative aversiveness of signalled vs unsignalled escapable and inescapable shock. Journal of the Experimental Analysis of Behavior, 17, $463-471$.

Berlyne, D. E. 1960 Conflict, arousal, and curiosity. New York: McGraw-Hill.

Bolles, R. C., \& Collier, A. C. 1976 The effect of predictive cues on freezing in rats. Animal Learning \& Behavior, 4, 6-8.

Collier, A. C. 1977 Preference for shock signals as a function of the temporal accuracy of the signals. Learning and Motivation, 8, $159-170$.

D’Amato, M. R., \& Safarjan, W. R. 1979 Preference for information about shock duration in rats. Animal Learning \& Behavior, 7, 89 - 94.

Fanselow, M. S. 1980 Signaled shock-free periods and preference for signaled shock. Journal of Experimental Psychology: Animal Behavior Processes, 6, 65-80.

Frankel, P. W., \& Vom Saal, W. 1976 Preference for predicted over unpredicted shock. Quar terly Journal of Experimental Psychology, 28, $441-447$.

Fujii, M., Uchida, M., \& Imada, H. 1994 Effects of presence and absence of information about shock intensity upon licking suppression in rats. Japanese Psychological Research, 36, 65 73.

Furedy, J. J., \& Biederman, G. B. 1976 Preference for signaled shock phenomenon: Direct and indirect evidence for modifiability factors in the shuttlebox. Animal Learning \& Behavior, $4,1-5$.

Imada, H., \& Okamura, M.1975Somecues rats can use as predictors of danger and safety. $A n i$ mal Learning \& Behavior, 3, 221 - 225.

Imada, H., \& Soga, M. 1971 The CER and BEL as a function of predictability and escapability of an electric shock. Japanese PsychologicalResearch, 13, $115-122$.

Imada, H., Sugioka, K., Ohki, Y., Ninohira, H., \& Yamazaki, A. 1978 The effects of double-alternation schedules of shock intensity upon patterning of suppression of licking in rats with special reference to cue-utilization. Japanese Psychological Research, 20, $167-176$.

Kawai, N., Nishida, N., \& Imada, H. 1998 Effects of postconditioning manipulations following compound conditioning on conditioned licking suppression in rats. Psychologia, 41, 49 59.

Lockard, J. S. 1963 Choice of a warning signal or no warning signal in an unavoidable shock situation. Journal of Comparative and Physiological Psychology, 56, $526-530$.

Marlin, N. A., Sullivan, J. M., Berk, A. M., \& Miller, R. R. 1979Preference for information about intensity of signaled tailshock. Learning and Motivation. 10, 85 - 97.

Nageishi, Y., \& Imada, H. 1974 Suppression of licking behavior in rats as a function of predictability of shock and probability of conditioned-stimulus-shock pairings.Journal of Comparative and Physiological Psychology, 87, $1165-1173$.

Perkins, C. C., Jr. 1968 An analysis of the concept of reinforcement. Psychological Review, 75, $155-172$.

Seligman, M. E. P. 1968 Chronic fear produced by unpredictable electric shock. Journal of $\mathrm{Com}$ parative and PhysiologicalPsychology, 66, 402 411.

Seligman, M. E. P., \& Meyer, B. 1970 Chronic fear and ulcers in rats as a function of the unpredictability of safety. Journal of Comparative and Physiological Psychology, 73, $202-207$.

Weiss,J.M.1970Somaticeffects of predictable and unpredictableshock.PsychosomaticMedicine, 32, $397-408$.

(Received Sept. 30, 1999; accepted Aug. 15, 2000) 